\title{
Direct interface for capacitive sensors based on the charge transfer method
}

\author{
Jorge E. Gaitán-Pitre ${ }^{1,2}$, Manel Gasulla ${ }^{2}$, and Ramon Pallàs-Areny ${ }^{2}$ \\ ${ }^{1}$ Universidad de Pamplona, Ciudadela Universitaria El Buque, Pamplona, Colombia \\ ${ }^{2}$ Instrumentation, Sensors and Interfaces Group, Castelldefels School of Technology (EPSC) \\ Technical University of Catalonia (UPC), Avda. del Canal Olímpic s/n, 08860 Castelldefels (Barcelona), Spain \\ Phone: +34-93-4137089, Fax: +34-93-4137007, Email: jorge.gaitan@ieee.org, ramon.pallas@upc.edu
}

\begin{abstract}
We present the theoretical analysis and experimental performance results of a direct interface for capacitive sensors based on the charge transfer method when parasitic capacitances are included. The interface circuit implements a two-point calibration technique that makes measurement results independent from voltage- and temperature-dependent parameters. The measurement deviation is below $1 \%$ FSR (Full Scale Range) for capacitances from $10 \mathrm{pF}$ to $1 \mathrm{nF}$.
\end{abstract}

Keywords - Direct sensor interface, capacitive sensor, charge transfer method, microcontroller, capacitance measurement.

\section{INTRODUCTION}

In recent years, some interface circuits for capacitive sensors have been implemented where the sensor is directly connected to a microcontroller (MCU). The MCU performs the capacitance-to-digital conversion without any external circuit for signal conditioning or analog-to-digital voltage conversion. Hence, these interface circuits are compact and inexpensive.

Direct capacitance-to-digital conversion relies on voltage comparison and timing. Reverter et al. [1] implemented a direct interface circuit based on measuring the discharging time of an $R C$ circuit that included the capacitive sensor. For a measurement range from $10 \mathrm{pF}$ to $100 \mathrm{pF}$, the measurement deviation was below $1.5 \%$ FSR. Nevertheless, to obtain the best speed-resolution trade-off when measuring in the picofarad range, they required a resistor larger than $10 \mathrm{M} \Omega$, which increased the sensitivity to noise and external interference. Hence, capacitive sensors with large electrodes cannot be easily measured with this method.

Dietz et al. [2] proposed an alternative circuit based on the charge transfer method, where the unknown capacitance is calculated by counting the number of charge transfer cycles needed to charge a reference capacitor to a threshold voltage via the capacitive sensor. In contrast with [1], the interface circuit did not include any resistor, so its susceptibility to noise and external interference should be lower. However, no quantitative information about circuit performance was provided.

Blake et al. [3] implemented a direct interface circuit that combined both techniques: the unknown capacitance was measured by the charge transfer method, and the circuit was calibrated by an $R C$ network with a known $R$. For capacitances below $300 \mathrm{pF}$, the measurement deviation was less than $10 \%$ FSR.
Whatever the method, direct interface circuits for capacitive sensors are sensitivity to parasitic capacitances, which increases the measurement uncertainty especially when measuring in the picofarad range. These parasitic capacitances mainly depend on the layout on the printed circuit board and on the wiring between sensor and the microcontroller, and their effects can be reduced by calibration [1].

This paper presents the theoretical analysis and experimental performance results of the direct interface circuit proposed in [2], when measuring in the range from $10 \mathrm{pF}$ to $1 \mathrm{nF}$ with a two-point calibration technique [4], and the effects of parasitic capacitance are included.

\section{INTERFACE CIRCUIT DESCRIPTION}

\section{A. Operating principle}

Figure 1 shows the operating principle for capacitance measurement based on the charge transfer method. It can be analyzed as a switched capacitor $R C$ circuit [5] where $C_{\mathrm{x}}$ is the unknown capacitance, $C_{\mathrm{r}}$ is the reference capacitance, and $V_{\mathrm{S}}$ the source voltage. All of them are assumed to be constant.

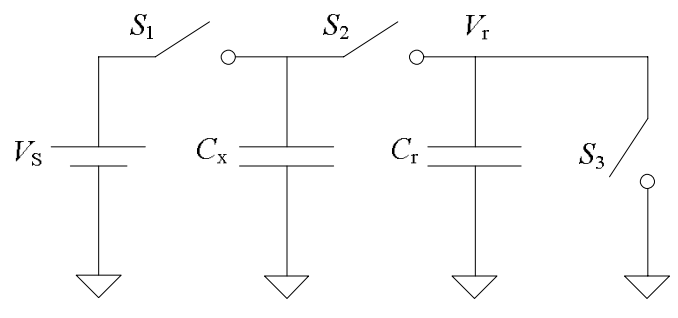

Fig. 1. Capacitance measurement circuit based on the charge transfer method.

The operation sequence is as follows. First, switch $S_{3}$ closes to reset $C_{\mathrm{r}}$. Then, $S_{3}$ opens and $S_{1}$ closes, and $C_{\mathrm{x}}$ is charged to $V_{\mathrm{S}}$. Next, $S_{1}$ opens and $S_{2}$ closes, so that the charge stored on $C_{\mathrm{x}}$ is shared with $C_{\mathrm{r}}$, which results in a voltage across $C_{\mathrm{r}}$ directly proportional to the charge transferred from $C_{\mathrm{x}}$ to $C_{\mathrm{r}}$. By repeating the charge transfer cycle, $C_{\mathrm{x}}$ exponentially charges $C_{\mathrm{r}}$ towards $V_{\mathrm{S}}$. This implies that $C_{\mathrm{r}}$ must be higher than $C_{\mathrm{x}}$. From here, $C_{\mathrm{x}}$ can be determined by counting the number of charge transfer cycles needed to charge $C_{\mathrm{r}}$ to a given threshold voltage $V_{\mathrm{T}}$. 
The voltage across $C_{\mathrm{r}}$ at any arbitrary $N$ charge transfer cycle is

$$
V_{\mathrm{r}}[N]=\frac{C_{\mathrm{x}}}{C_{\mathrm{x}}+C_{\mathrm{r}}} V_{\mathrm{S}}+\frac{C_{\mathrm{r}}}{C_{\mathrm{x}}+C_{\mathrm{r}}} V_{\mathrm{r}}[N-1]
$$

If we assume $V_{\mathrm{r}}[0]=0$ because of the initial closing of $S_{3}$, by summing the geometric progression we obtain,

$$
V_{\mathrm{r}}[N]=V_{\mathrm{S}}\left[1-\left(\frac{C_{\mathrm{r}}}{C_{\mathrm{x}}+C_{\mathrm{r}}}\right)^{N}\right]
$$

From here, considering $C_{\mathrm{r}}>>C_{\mathrm{x}}$, we obtain

$$
N=-\frac{1}{C_{\mathrm{x}}} C_{\mathrm{r}} \ln \left(1-\frac{V_{\mathrm{T}}}{V_{\mathrm{S}}}\right)
$$

that can be rearranged as

$$
C_{\mathrm{x}}=\frac{k}{N}
$$

where $k=-C_{\mathrm{r}} \ln \left(1-V_{\mathrm{T}} / V_{\mathrm{S}}\right)$. From (3), $N$ increases at a rate proportional to $C_{\mathrm{r}} / C_{\mathrm{x}}$.

\section{B. Interface circuit}

Figure 2 shows the interface circuit proposed in [2] plus parasitic capacitances to ground $C_{\mathrm{p} 0}$ and $C_{\mathrm{p} 1} . C_{\mathrm{x}}$ is the capacitance to be measured; $C_{\mathrm{r}}$ is the reference capacitor; $C_{\mathrm{p} 0}$ and $C_{\mathrm{p} 1}$ model the parasitic capacitances from nodes 0 and 1 to ground, respectively; $R_{\mathrm{OL}}$ and $R_{\mathrm{OH}}$ model the channel resistance of the transistor of the output buffer when it provides a digital " 0 " and a digital " 1 ", respectively; the values of this resistances can be assumed constant whenever the transistor works in its ohmic region [6]. $V_{\mathrm{OL}}$ and $V_{\mathrm{OH}}$ are the output voltage levels when it provides a digital " 0 " and a digital "1" respectively.

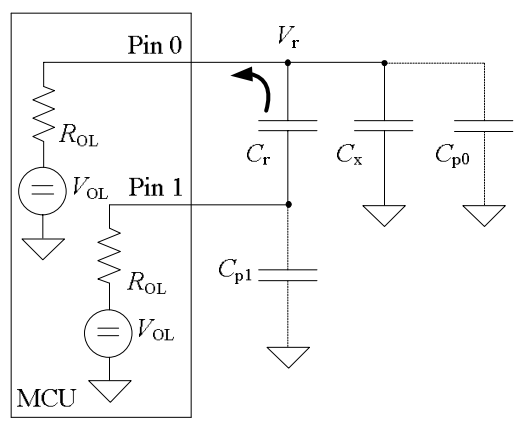

(a)

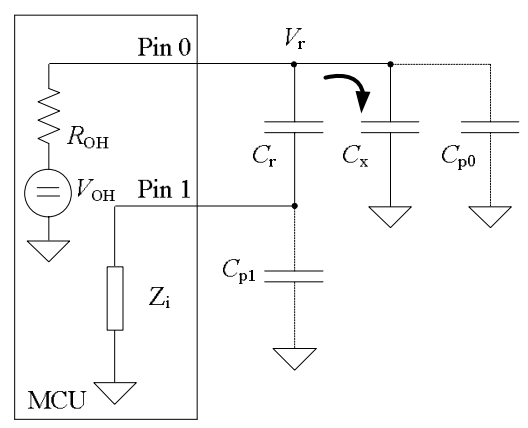

(b)

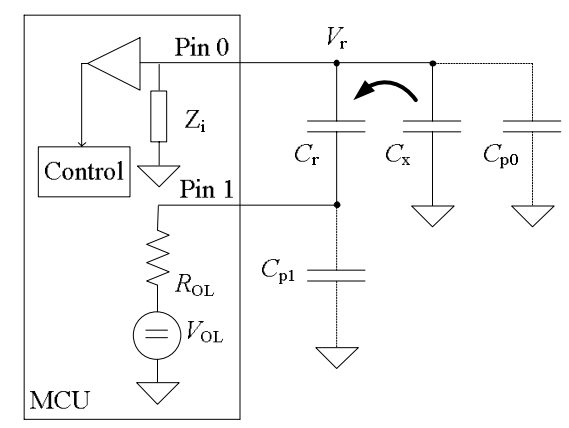

(c)

Fig. 2. Direct interface for a capacitive sensor based on the charge transfer method. (a) Resetting stage (only at the beginning of the measurement). (b) Charging stage. (c) Transferring stage. 


\section{Calibration technique}

Calibration makes measurement results independent from $V_{\mathrm{OH}}, V_{\mathrm{T}}$, and $C_{\mathrm{r}}$, hence from $k$. Because stray capacitances add a zero effect to the transfer characteristic in (6), we can also expect to reduce that effect by calibration. So, a twopoint calibration technique can compensate for these two effects.

Figure 3 shows the proposed interface circuit with two calibration points. Only parasitic capacitances to ground have been included, because use to be the larger stray capacitances. This calibration technique implies three measurements, one for each reference capacitor $\left(C_{\mathrm{c} 1}, C_{\mathrm{c} 2}\right)$ and one for the unknown capacitance $\left(C_{\mathrm{x}}\right)$.

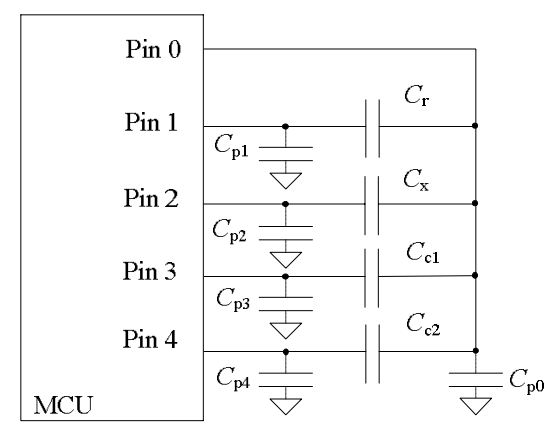

Fig. 3. Interface circuit to implement a two-point calibration technique when parasitic capacitances to ground are included.

Applying to each capacitor the method described in the Section II.B, we obtain

$$
\begin{aligned}
& C_{\mathrm{x}}=\frac{k}{N_{\mathrm{x}}}-C_{\mathrm{eq}-\mathrm{x}} \\
& C_{\mathrm{c} 1}=\frac{k}{N_{\mathrm{c} 1}}-C_{\mathrm{eq}-\mathrm{c} 1} \\
& C_{\mathrm{c} 2}=\frac{k}{N_{\mathrm{c} 2}}-C_{\mathrm{eq}-\mathrm{c} 2}
\end{aligned}
$$

where $N_{\mathrm{x}}, N_{\mathrm{c} 1}$, and $N_{\mathrm{c} 2}$ are the number of charge transfer cycles for, respectively: (a) the unknown capacitance $C_{\mathrm{x}}$, (b) the first reference capacitor $C_{\mathrm{cl}}$, and (c) the second reference capacitor $C_{\mathrm{c} 2} ; C_{\mathrm{eq}-\mathrm{x}}, C_{\mathrm{eq}-\mathrm{c} 1}$, and $C_{\mathrm{eq}-\mathrm{c} 2}$ model the equivalent parasitic capacitances when measuring $N_{\mathrm{x}}, N_{\mathrm{c} 1}$, and $N_{\mathrm{c} 2}$, respectively. If we assume that $k$ remains the same over the time for the three measurements and $C_{\text {eq-x }} \approx C_{\text {eq-c1 }} \approx C_{\text {eq-c2 }}$, solving (7) for $C_{\mathrm{x}}$, yields

$$
C_{\mathrm{x}}=\frac{1}{N_{\mathrm{x}}} \frac{N_{\mathrm{c} 1} N_{\mathrm{c} 2}}{N_{\mathrm{c} 1}-N_{\mathrm{c} 2}}\left(C_{\mathrm{c} 2}-C_{\mathrm{c} 1}\right)-\frac{N_{\mathrm{c} 2} C_{\mathrm{c} 2}-N_{\mathrm{c} 1} C_{\mathrm{c} 1}}{N_{\mathrm{c} 1}-N_{\mathrm{c} 2}}
$$

which is independent from $k$ and from the parasitic capacitances. By comparing with (6) we see that gain uncertainty has been reduced because the gain now depends on known parameters, and that offset effects have been reduced because they do not include any stray capacitance.

\section{MATERIALS AND METHOD}

The circuit interface proposed in Fig. 3 has been implemented by using a PIC16F84A MCU, operating at $4 \mathrm{MHz}$ in crystal oscillator mode. The function of pins $0,1,2$, 3 and 4 were implemented by pins RB0, RB1, RB3, RB4 and RB7 respectively. The control program was written in assembler language. The instruction cycle time was $1 \mu \mathrm{s}$, one-fourth of the main oscillation period.

The proposed interface circuit was applied to measure capacitors from $10 \mathrm{pF}$ to $1 \mathrm{nF}$. All of them were ceramic capacitors with $10 \%$ tolerance. $C_{\mathrm{r}}$ was $1 \mu \mathrm{F} \pm 0.1 \mu \mathrm{F}$ with metallized polyester dielectric. Actual $C_{\mathrm{x}}$ and $C_{\mathrm{r}}$ values were measured by an impedance analyzer (Agilent 4294A) connected to a test fixture (Agilent 16047E). The basic uncertainty of the impedance analyzer is below $1 \%$ in the measurement range from $10 \mathrm{pF}$ to $1 \mathrm{nF}$, when measuring at $100 \mathrm{kHz}$ and $0.5 \mathrm{~V}$ (rms oscillator output level) [8]. $T_{\mathrm{D}}, T_{\mathrm{C}}$ and $T_{\mathrm{R}}$ were calculated via $R_{\mathrm{OL}}$ and $R_{\mathrm{OH}}$ for pins $\mathrm{RB} 0, \mathrm{RB} 1$, $\mathrm{RB} 3$, RB4, and RB7, which were indirectly measured by the voltage-divider technique describe in [6].

The interface circuit was evaluated for two measurement sub-ranges: from $10 \mathrm{pF}$ to $100 \mathrm{pF}$, and from $100 \mathrm{pF}$ to $1 \mathrm{nF}$. For $C_{\mathrm{c} 1}$ and $C_{\mathrm{c} 2}$ we selected the end values of each sub-range. For each capacitor $C_{\mathrm{x}}, C_{\mathrm{c} 1}$, and $C_{\mathrm{c} 2}$, the MCU consecutively measured $N_{\mathrm{x}}, N_{\mathrm{c} 1}$, and $N_{\mathrm{c} 2} 100$ times. The values obtained were sent to a personal computer via a serial link (EIA-232) implemented with a MAX233 under LabVIEW control. Then, 100 values of $C_{\mathrm{x}}$ were calculated by applying (8) and its mean value was calculated. The measurement accuracy was evaluated as the deviation of the mean value from the actual value.

To design the circuit interface the following guidelines were followed:

1.To avoid large parasitic capacitances, $C_{\mathrm{x}}, C_{\mathrm{c} 1}$ and $C_{\mathrm{c} 2}$ were placed as close as feasible to the MCU.

2.To reduce the effects of external interference, the unused $\mathrm{I} / \mathrm{O}$ pins of the MCU were configured as inputs and connected to ground. Further, the printed circuit board did not have any ground plane.

3.To reduce the effects of power supply noise, the MCU and MAX233 were supplied by two independent voltage regulators (LM7805). Moreover, a decoupling capacitor $C_{\mathrm{d}}$ $=100 \mathrm{nF}$ was connected between the MCU power supply pin and ground [6].

\section{IV.EXPERIMENTAL RESULTS AND DISCUSSION}

The interface circuit in Fig. 3 was first characterized by measuring some of its parameters according to the procedure 
described in Section III. The experimental results were as follows. The internal output resistance for pins RB0, RB1, $\mathrm{RB} 3, \mathrm{RB} 4$ and RB7, when they provide a digital "0" or a digital " 1 ", were below $50 \Omega$ and $125 \Omega$, respectively. $C_{\mathrm{x},-\max }$ and $C_{\mathrm{r}}$ were $0.96 \mathrm{nF}$ and $1.06 \mu \mathrm{F}$, respectively. Consequently, $T_{\mathrm{D}}, T_{\mathrm{C}}$ and $T_{\mathrm{R}}$ should be larger than $0.53 \mathrm{~ms}, 1.2 \mu \mathrm{s}$ and $0.48 \mu \mathrm{s}$, respectively. However, we selected $T_{\mathrm{D}}=1 \mathrm{~s}$ to reduce any possible dielectric absorption effects in $C_{\mathrm{r}}$ [7]. $T_{\mathrm{C}}$ and $T_{\mathrm{R}}$ were selected $5 \mu \mathrm{s}$ and $25 \mu \mathrm{s}$, respectively, by considering the minimal number of instructions to execute at each stage. Figure 4 shows the measurement deviation achieved for each of the ten measured capacitors, which is below $1 \%$ FSR for $10 \mathrm{pF}<C_{\mathrm{x}}<1 \mathrm{nF}$. These deviations are acceptable for many industrial applications and are smaller than those reported in the bibliography for other direct interface circuits. Moreover, they were obtained without shielding the circuit, which means that the measured sensors can have large dimension, e.g. strip electrodes.

\section{CONCLUSIONS}

The charge method offers a simple, compact, and low-cost solution to directly connect capacitive sensors to microcontrollers. A theoretical analysis shows the relevant MCU parameters involved, and the effects of parasitic capacitances on the transfer characteristic. A calibration circuit with two known capacitors reduces these effects. The interface circuit has been evaluated by using a low cost MCU (PIC16F84A) and applying some design guidelines. For capacitors from $10 \mathrm{pF}$ to $1 \mathrm{nF}$, the measurement deviation was below $1 \% \mathrm{FSR}$. This value is acceptable for many applications and it was obtained without any circuit shielding.

\section{APPENDIX}

Referring to Fig. $2 b$, at any arbitrary charge transfer cycle, the charge stored on the parallel combination of $C_{\mathrm{x}}$ and $C_{\mathrm{p} 0}$ is

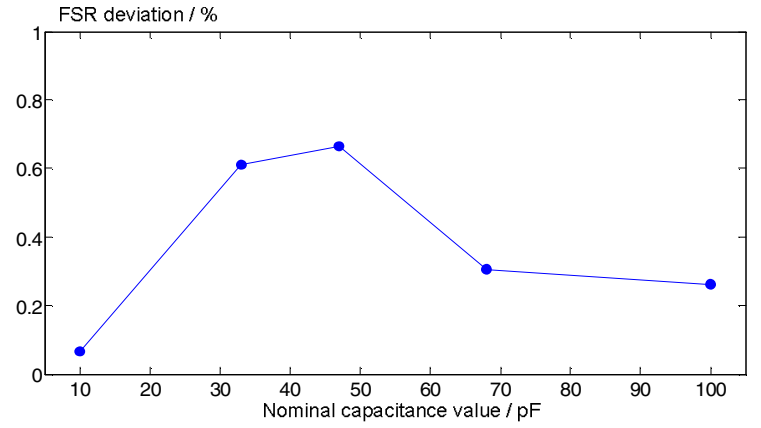

(a)

$$
Q_{\mathrm{x}}[N-1 / 2]=V_{\mathrm{OH}}\left(C_{\mathrm{x}}+C_{\mathrm{p} 0}\right)
$$

In this same stage, the combination of $C_{\mathrm{r}}$ and $C_{\mathrm{p} 01}$ is also charged. This charge is

$$
Q_{\mathrm{r}}[N-1 / 2]=V_{\mathrm{r}}[N-1 / 2] C_{\mathrm{r}}
$$

where $V_{\mathrm{r}}[N-1 / 2]$ is

$$
V_{\mathrm{r}}[N-1 / 2]=\frac{C_{\mathrm{r}}}{C_{\mathrm{r}}+C_{\mathrm{p} 1}} V_{\mathrm{r}}[N-1]+\frac{C_{\mathrm{p} 1}}{C_{\mathrm{r}}+C_{\mathrm{p} 1}} V_{\mathrm{OH}}
$$

During the transferring stage, $Q_{\mathrm{x}}[N-1 / 2]$ and $Q_{\mathrm{r}}[N-1 / 2]$ are shared. So, the voltage across $C_{\mathrm{r}}$ at the end of charge transfer cycle is

$$
V_{\mathrm{r}}[N]=\frac{Q_{\mathrm{x}}[N-1 / 2]+Q_{\mathrm{r}}[N-1 / 2]}{C_{\mathrm{x}}+C_{\mathrm{p} 0}+C_{\mathrm{r}}}
$$

Substituting (A1) and (A2) in (A4), we obtain

$$
\begin{aligned}
V_{\mathrm{r}}[N]= & \frac{V_{\mathrm{OH}}}{C_{\mathrm{x}}+C_{\mathrm{p} 0}+C_{\mathrm{r}}}\left(C_{\mathrm{x}}+C_{\mathrm{p} 0}+\frac{C_{\mathrm{r}} C_{\mathrm{p} 1}}{C_{\mathrm{r}}+C_{\mathrm{p} 1}}\right) \\
& +\frac{C_{\mathrm{r}}^{2}}{\left(C_{\mathrm{x}}+C_{\mathrm{p} 0}+C_{\mathrm{r}}\right)\left(C_{\mathrm{r}}+C_{\mathrm{p} 1}\right)} V_{\mathrm{r}}[N-1]
\end{aligned}
$$

Then, applying sum of geometric progression and assuming $V_{\mathrm{r}}[0]=0$ we obtain

$$
V_{\mathrm{r}}[N]=V_{\mathrm{OH}}\left\{1-\left[\left(\frac{C_{\mathrm{r}}}{C_{\mathrm{x}}+C_{\mathrm{p} 0}+C_{\mathrm{r}}}\right)\left(\frac{C_{\mathrm{r}}}{C_{\mathrm{r}}+C_{\mathrm{p} 1}}\right)\right]^{N}\right\}
$$

Fig. 4. FSR deviation for measured capacitance from: (a) $10 \mathrm{pF}$ to $100 \mathrm{pF}$, and (b) $100 \mathrm{pF}$ to $1 \mathrm{nF}$. 


\section{ACKNOWLEDGMENTS}

This work has been funded by the Spanish Ministry of Education and Science under contract TEC2004-05520 and by the European Regional Development Fund. The authors appreciate the discussions and insight provided by Oscar Casas and the technical support of Francis López. Jorge E. Gaitán-Pitre is partially supported by the University of Pamplona.

\section{REFERENCES}

[1] F. Reverter, M. Gasulla, R. Pallàs-Areny, "A Low-Cost Microcontroller Interface for Low-Value Capacitive Sensors.", Proc IEEE Instrumentation and Measurement Technology Conference 2004, Como, Italy, 18-20 May 2004, pp. 1771-1775.
[2] P. H. Dietz, D. Leigh, W. S. Yerazunis, "Wireless Liquid Level Sensing for Restaurant Applications.", Proc. IEEE Sensors 2002, pp. 715-719.

[3] K. Blake, S. Bible. "Measuring Small Changes in Capacitive Sensors." AN1014, Microchip Technology Inc. Application Note 2005.

[4] R. Pallàs-Areny and J. G. Webster, "Sensors and Signal Conditioning." New York: 2nd ed., John Wiley \& Sons, 2001.

[5] P. E. Allen, E. Sánchez-Sinencio. "Switched Capacitor Circuits". Van Nostrand-Reinhold, New York: 1984.

[6] F. Reverter, J. Jordana, M. Gasulla and R. Pallàs-Areny, "Accuracy and Resolution of Direct Resistive Sensor-to-Microcontroller Interfaces." Sensors and Actuators A, Vol. 121, pp. 78-87, Jan. 2005.

[7] Kuenen, J.C.; Meijer, G.C.M., "Measurement of Dielectric Absorption of Capacitors and Analysis of its Effects on VCOs," IEEE Trans. on Instrum. Meas., Vol. 45, No.1, pp. 89-97, Feb. 1996.

[8] Agilent Technologies. Agilent 4294A Precision Impedance Analyzer. Data Sheet 2003. 